\title{
Adaptive Synergetic Controller for Stabilizing the Altitude and Angle of Mini Helicopter
}

\author{
ZAIN ANWAR ALI*, AND LI XINDE* \\ RECEIVED ON 12.08.2018 ACCEPTED ON 19.02.2019
}

\begin{abstract}
This research proposes ASC (Adaptive Synergetic Controller) for the nonlinear model of MH (Mini Helicopter) to stabilize the desired altitude and angle. The model of MH is highly nonlinear, underactuated and multivariable in nature due to its dynamic uncertainties and restrictions of velocities during the flight. ASC can force the tracking errors of the system states converges to zero in a finite interval of time. The MH system requires smooth controller and fast precise transition response from initial state till the desired state, therefore the parametric calculations and simulations can be done by the proposed ASC algorithm. It is validated that the above simulated results of the proposed controller have a better convergence rate and smoother stability response in order to track the desired altitude and angle when compared with SMC (Sliding Mode Controller). Moreover, it does not need any linearization, transformation and variations in the system model.
\end{abstract}

Key Words: Adaptive Control, Synergetic Controller, Sliding Mode Control, Mini Helicopter, Control Algorithm and Adaptive Synergetic Control.

\section{INTRODUCTION}

$\Gamma$

his article presents the modelling, controlling and its simulation of X-Cell (50) Yamaha MH. It is established on the bases of differential and state space mathematical model [1-3]. Adaptive controller based synergetic control algorithm is designed for stabilizing the desired or referred altitude and angle of MH is designed. Moreover, the synergetic control scheme is capable to update the dynamic performance (heading angle and altitude) of the system and complete system gains are fine-tuned by adaptive controller. Despite the other rotor planes, MH has complex dynamics due to its under-actuated, non-holonomic behavior in nature [4].
Formerly, many researchers, scientists pay attention on the linearization and stabilization of $\mathrm{MH}$, because of its nonlinear dynamics [5].

For proper controlling, stabilizing altitude and heading angle of helicopter initially we require the stability of its nonlinear dynamics at different points commonly called as trim point conditions in control engineering [6-8]. Secondly, it may concern the referred position, velocity constraints and desired orientation to track given path. The controller designing of MH is more complex than general aircraft in terms of coupling between the lateral 
channels and longitudinal parameters. Simultaneously the other constraints like hysteresis effect, unwanted disturbance and highly nonlinearity increase the complexity in the designing of controller [9-10].

Previously, different linear and nonlinear control approaches were designed to control the dynamics of MH [11]. However, the main disadvantages of the previous algorithms are the multiple constraints, inherent complexity of the system parameters and low performance. To manage the aforementioned drawbacks, this research focus on nonlinear MIMO (Multiple Input Multiple Output) system based on adaptive control via synergetic controller. It is very difficult to achieve the equilibrium state in the presence of steady state errors and dynamic coupling technique [12-15]. The traditional method for equilibrium to overcome the disturbance is not enough, therefore a strong synergistic control method must be designed to control the MIMO based system. In [16-19], the same model of MH X-Cell 50 was used to ensure the steady hovering or stability of the proposed nonlinear autopilot controller, such that the nonlinear system stability was done by the Lyapunov direct method. The Lyapunov direct method does not require linearization of system states transformation.

The designed scheme also guarantees the better stability over other conventional methods and provides faster system response for equilibrium even in the presence of nonlinear uncertainties. Recently the advancement in the control theory, algorithm for the controlling of nonlinear system has been remarkable and the complexities of the helicopter are handled more mannerly such as dynamic inversion method, synergetic control method, etc. [2024]. The results and simulations of the proposed scheme validate the better stability response when compared with SMC.
In this research, the performance of our proposed ASC is compared with conventional SMC. The major contributions of this research are: (1) a new ASC is designed which is able to handle the uncertainty and auto tuning of control parameters; (2) the proposed controller uses two control input commands as macro variables for controlling and stabilizing the altitude and heading angle of helicopter; (3) the 5th degree (higher degree) nonlinear equation of helicopter is become controllable by applying the Chow's theory; (4) the convergence rate of the synergetic controller provides the helicopter model to the desired altitude at short interval of time.

The breakup of this manuscript is structured as shown. Section 2 defines the structure of MH. It is followed by the designing of the proposed controller in section 3 . Section 4 discusses the convergence rate of the desired performance. The simulation result defines in section 5, which shows the robustness and desired performance of the system. Finally, section 6 outlines the conclusions.

\section{SYSTEM MODEL}

The basic helicopter model consists of four inputs called as lateral, longitudinal, pedal and collective. Two velocity components are angular, linear denoted as (p,q,r), (u,v,w), Euler angles $(\varphi, \vartheta, \psi)$ and its movements are $(\mathrm{x}, \mathrm{y}, \mathrm{z})$ axis respectively, such that ' $z$ ' axis shows its altitude [25]. In this article we only consider two inputs of the MIMO model of $\mathrm{MH}$ and check their responses by tuning the system dynamics as per our desired altitude and collective pitch angle.

The following system model shown in Fig. 1 is taken from [18], Equations (1-2) show the differential equations model in which vertical altitude and rotational speed of rotors are written as, 
$z=K_{1} C_{1} \delta^{2}-g-K_{2} z-K_{3} \dot{z}^{2}-K_{4}$

$\left\{\begin{array}{l}C_{t}=\left[K_{C 1}+\left(K_{C 1}^{2}+K_{C 2} \vartheta\right)^{\frac{1}{2}}\right]^{2} \\ \dot{\delta}=-K_{5} \delta-K_{6} \delta^{2}-K_{1} \delta^{2} * \sin \vartheta+K_{8} \mu_{1}+K_{9} \\ \ddot{\vartheta}_{c}=K_{10}\left(-3.174 * 10^{-4} * \mu_{2}+0.5436-\vartheta\right)-K_{11} \dot{\vartheta}-K_{12} \delta^{2} \sin \vartheta\end{array}\right.$

Where altitude or height of helicopter is denoted by ' $z$ ' in meters that is above the ground level, the rotational

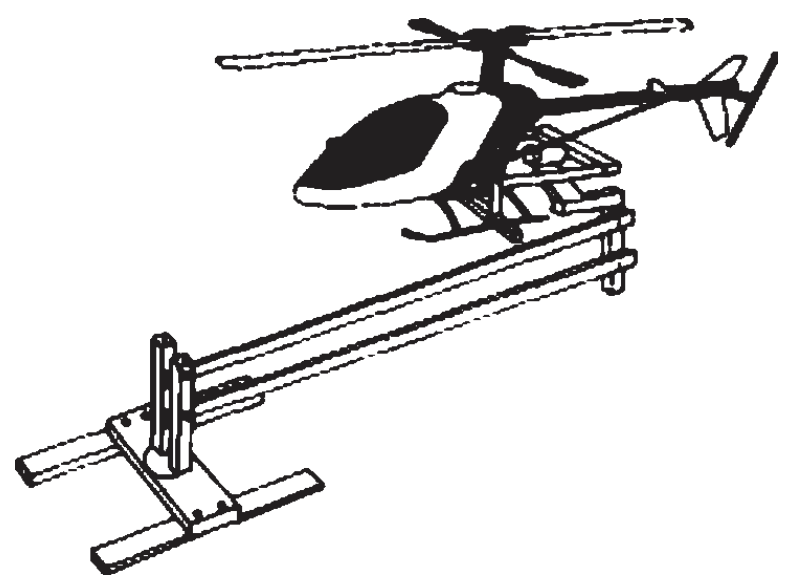

FIG. 1. MODEL OF YAMAHA X-CELL $50 \mathrm{MH}$ speed of rotors is defined by ' $\delta$ ' $(\mathrm{rad} / \mathrm{s})$, gravitational force is ' $\mathrm{g}$ ' $\left(\mathrm{m} / \mathrm{s}^{2}\right)$, ' $\mathrm{v}_{\mathrm{c}}$ ' is the collective angle of pitch and ' $\mathrm{C}_{\mathrm{t}}$ ' is the coefficient of thrust. Where $\mu_{1}, \mu_{2}$ are the throttle and collective inputs which is utilized to control the altitude and angle of MH. The marginal values of the system constraints are taken from [17-19] are described in Table 1.

Fig. 2 represents the heading angle, angular rotation and the desired height of MH. The differential Equations (12) are now converted into state space matrix which is defined in Equations (3-6), where the altitude of $\mathrm{MH}$ varies with angle and at times the gravitational effects in the system in neglected [18].

$\left\{\begin{array}{c}\dot{x}=\xi(x)+\zeta_{1}(x) \mu_{1}+\zeta_{2}(x) \mu_{2} \\ y=\left[y_{1} y_{2}\right]^{T}=\left[x_{1} x_{2}\right]^{T}\end{array}\right.$

Where the input control variables and the system states are defined by:

TABLE 1. YAMAHA X-CELL 50 MODEL CONSTANTS

\begin{tabular}{|c|c|c|c|c|}
\hline Constants & Value & Unit & Constants & Value \\
\hline $\mathrm{g}$ & 9.8 & $\mathrm{~m} / \mathrm{s}^{2}$ & $\mathrm{~K}_{\mathrm{C} 2}$ & $6.1456^{*} 10^{-2}$ \\
\hline $\mathrm{K}_{1}$ & $25^{*} 10^{-2}$ & $\mathrm{M}$ & $\mathrm{a}_{1}$ & $53.1^{*} 10^{-3}$ \\
\hline $\mathrm{K}_{2}$ & $10^{*} 10^{-2}$ & $1 / \mathrm{s}$ & $\mathrm{a}_{2}$ & $15.364 * 10^{-3}$ \\
\hline $\mathrm{K}_{3}$ & $10^{*} 10^{-2}$ & $1 / \mathrm{m}$ & $\mathrm{a}_{3}$ & $282 * 10^{-9}$ \\
\hline $\mathrm{K}_{4}$ & 7.86 & $\mathrm{~m} / \mathrm{s}^{2}$ & $\mathrm{a}_{4}$ & $163.2 * 10^{-6}$ \\
\hline $\mathrm{K}_{5}$ & $70^{*} 10^{-2}$ & $1 / \mathrm{s}$ & $-\mathrm{K}_{2}$ \\
\hline $\mathrm{K}_{6}$ & $28^{*} 10^{-4}$ & - & $\mathrm{a}_{6}$ & $-\mathrm{K}_{2}$ \\
\hline $\mathrm{K}_{7}$ & $5 * 10^{-3}$ & - & $\mathrm{a}_{7}$ & $-\mathrm{g}^{*} \mathrm{~K}_{4}$ \\
\hline $\mathrm{K}_{8}$ & $10.88^{*} 10^{-2}$ & $1 / \mathrm{s}^{2}$ & $\mathrm{a}_{8}$ & $-\mathrm{K}_{5}$ \\
\hline $\mathrm{K}_{9}$ & -13.92 & $1 / \mathrm{s}^{2}$ & $\mathrm{a}_{9}$ & $-\mathrm{K}_{6}$ \\
\hline $\mathrm{K}_{10}$ & 800.0 & $1 / \mathrm{s}^{2}$ & $\mathrm{a}_{10}=\mathrm{a}_{14}$ & $-\mathrm{K}_{6}$ \\
\hline $\mathrm{K}_{11}$ & 65.0 & $1 / \mathrm{s}$ & $\mathrm{a}_{11}$ & $\mathrm{~K}_{9}$ \\
\hline $\mathrm{K}_{12}$ & 0.1 & - & $\mathrm{a}_{12}$ & $0.5436^{*} \mathrm{~K}_{10}$ \\
\hline $\mathrm{KC}_{1}$ & $32.59 * 10^{-3}$ & - & $\mathrm{a}_{3}$ & $-\mathrm{K}_{10}$ \\
\hline
\end{tabular}


$\xi(x)=\left\{\begin{array}{c}\mu=\left[\mu_{1} \mu_{2}\right]^{T}=\left[K_{8} * \mu_{1}-3.176^{*} 10^{-3} * \mu_{2} * K_{10}\right] \\ x=\left[x_{1} x_{2} x_{3} x_{4} x_{5}\right]^{T}=[z \dot{z} \delta \vartheta \dot{\vartheta}] \\ \xi(x)\left[\xi_{1}(x), \xi_{2}(x), \xi_{3}(x), \xi_{4}(x), \xi_{5}(x)\right] \\ \zeta_{1}(x)=\left[\begin{array}{l}0 \\ 0 \\ 1 \\ 0 \\ 0\end{array}\right] \zeta_{2}(x)=\left[\begin{array}{l}0 \\ 0 \\ 0 \\ 0 \\ 1\end{array}\right]\end{array}\right.$

$\xi(x)=\left\{\begin{array}{c}x_{2} \\ a_{0}+a_{1} x_{2}+a_{2} x_{2}^{2}+\left(a_{3}+a_{4} x_{4}-\sqrt{a_{5}+a_{6} x_{4}}\right) x_{3}^{2} \\ a_{7}+a_{8} x_{3}+\left(a_{9} \sin \left(x_{4}\right)+a_{10}\right) x_{3}^{2} \\ x_{5} \\ a_{11}+a_{12} x_{4}-a_{13} x_{3}^{2} \sin \left(x_{4}\right)+a_{14} x_{5}\end{array}\right.$

$\xi(x)=\left\{\begin{array}{c}\dot{x}_{1}=\xi_{1} x_{2} \\ \dot{x}_{2}=\xi_{2}=a_{0}+a_{1} x_{2}+a_{2} x_{2}^{2}+\left(a_{3}+a_{4} x_{4}-\sqrt{a_{5}+a_{6} x_{4}}\right) x_{3}^{2} \\ \dot{x}_{3}=\xi_{3}+\mu_{1}=a_{7}+a_{8} x_{3}+\left(a_{9} \sin \left(x_{4}\right)+a_{10}\right) x_{3}^{2} \\ \dot{x}_{4}=\xi_{4}=x_{5} \\ \dot{x}_{5}=\xi_{5}+\mu_{2}=a_{11}+a_{12} x_{4}+a_{13} x_{3}^{2} \sin \left(x_{4}\right)+a_{14} x_{5}\end{array}\right.$

There are two couplings in the dynamics of system states and the second is the control input (operational coupling). In the following section, nonlinear model of the system is controlled by the ASC strategy to control the dynamics of the system and the results presented in this research. Lie bracket is represented by $[\xi(\mathbf{x}), \zeta(\mathbf{x})]$ of functions $\xi(\mathbf{x})$ and $\zeta_{1}(\mathbf{x})$.

$\left[\xi(x), \zeta_{1}(x)\right]=\frac{\partial \xi(x)}{\partial x} \xi_{1}(x)-\frac{\partial \xi_{1}(x)}{\partial x} \xi(x)$

Similarly Lie Bracket of $\xi(\mathbf{x}), \zeta(\mathbf{x})$ and $\zeta_{2}(\mathbf{x})$ is given by:

$$
\left[\xi(x), \zeta_{1}(x)\right]=\left[\begin{array}{c}
0 \\
2 x_{3}\left(a_{3}+a_{4} x_{4}-\sqrt{a_{5}+a_{6} x_{4}}\right) \\
2 x_{3}\left(a_{9} \sin \left(x_{4}\right)+a_{10}\right) \\
0 \\
2 a_{13} x_{3} \sin \left(x_{4}\right)
\end{array}\right]
$$

$$
\left[\xi(x), \zeta_{2}(x)\right]=\frac{\partial \xi(x)}{\partial x} \zeta_{2}(x)-\frac{\partial \zeta_{2}(x)}{\partial x} \xi(x)=\left[\begin{array}{c}
0 \\
0 \\
0 \\
1 \\
a_{14}
\end{array}\right]
$$

Now, according to the Chow's theory, if the non-linear system is in the form of $\dot{x}=\dot{\xi}(\mathrm{x})+\zeta_{1} \mu_{1}+\zeta_{2} \mu_{\mathrm{u}}+\ldots+\zeta_{\mathrm{m}} \mu_{\mathrm{m}}$; having 5 th order system matrix. It must be controllable using involute closure of set $\left\{\xi, \zeta_{1}, \ldots, \zeta_{2}\right\}$ [26]. However, if $\mathbf{x}_{2}, \mathbf{x}_{3}$ are non-zero then the above matrix is off 5 th order because of the rotational speed and height convergence to zero.

\section{SYNERGETIC CONTROLLER}

Nonlinear systems are complex in nature, they can be controlled by adaptive, robust and synergetic control theory based on an analytical approach of aggregation [27]. The designing process of the controller begins by identifying the micro variable. In this research, an algorithm is proposed that follows an adaptive synergetic controlled which is the main contribution of this research [28]. In order to enhance the robust control design, including a continuous control law and finite time convergence of the errors in a fully non-linear system simulation [29]. The system dynamics of synergetic control along with attractors $\psi_{\mathrm{i}}=0$ are shown by:

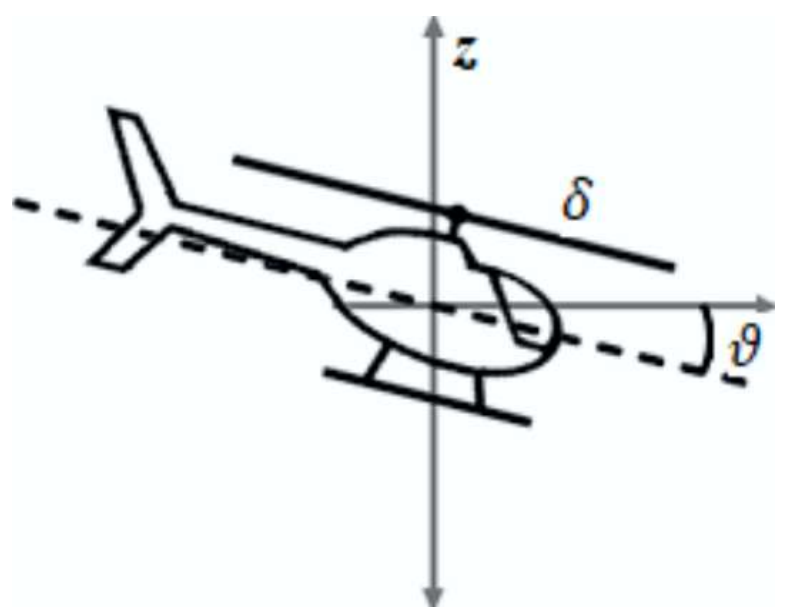

FIG. 2. THE MODEL OF $M H$ 
$\mathrm{T} \dot{\rho}+\beta(\rho)=0$

Where the convergence rate represented by $\mathrm{T}, \psi$ is the time derivative of macro variable and reaching effect function $\beta($.) which is also equal to $\beta(\rho)=\rho$ Therefore the Equation (10) can be written as:

$$
\mathrm{T} \dot{\rho}+\rho=0
$$

The above differential equation is evaluated by:

$$
\rho(t)=\rho_{0} e^{-\frac{t}{T}}
$$

Where ' $t$ ' is the time and ' $T$ ' is the convergence rate as shown above. Transient speed of the controller can be enhanced by varying ' $T$ ' inversely [30]. In the coming section, the algorithm can be made using the core parameters of synergetic controller for obtaining the roots of the expressions. The nonlinear equation of the system is already defined in Equation (3).

\section{CONVERGENCE RATE}

In accordance with dynamic Equation (10) $\mathrm{T} \dot{\rho}+\rho=0$, $\rho$ represented by $\rho_{0}$ at time $t_{0}$ and the simplification of the above expression is given by:

$$
\rho_{i}(t)=\rho_{i}\left(t_{0}\right) e^{-\left(\frac{t_{0}}{T_{i}}\right)}
$$

Here $\rho_{\mathrm{t}}$ is inversely proportional to the time $t$ i.e. as the time approaches to infinity it becomes zero, therefore the movement of the system state from the initial state to final state is possible. Furthermore, the $\mathrm{T}_{\mathrm{i}}$ in the above equation represent the convergence rate of the state variable to the final stage. If somehow the system variable is stable and the $T_{i}$ remains smaller, the convergence rate is faster to achieve its target. The desired output utilizes the shorter process and give better result. Where $\mathrm{e}_{1}, \mathrm{e}_{2}$ are the error of altitude, heading angle and $\mathrm{c}_{\mathrm{ii}}$ are the constants of controller that has minor value less than (0.1).

$\left\{\begin{array}{c}\rho_{1}=c_{11} e_{1}+c_{12} e_{1}+e_{1} \\ \rho_{2}=c_{21} e_{2}+e_{2}\end{array}\right.$

Where $\mathrm{e}_{1}=\mathrm{i}_{1}-\mathrm{x}_{1}$ and $\mathrm{e}_{2}=\mathrm{i}_{2}-\mathrm{x}_{3}$.

Considering $i_{1}$ is desired altitude, $i_{2}$ is desired angle, $\mu_{1}$ and $\mu_{2}$ are macro variable control signals of dynamical Equation (15).

$\mathrm{T}_{1} \mathrm{r}_{1}+\mathrm{r}_{1}=0$

Where $\rho_{1}=c_{11} e_{1}+c_{12} e_{1}+e_{1}, e_{1}=-x_{1}=-x_{2}, e_{1}=x_{2}=-\xi_{2}$ and $e_{1}=-\xi_{2}$.

The combined equation is given by:

$\mathrm{T}_{1} \mathrm{c}_{11} \mathrm{e}_{1}+\mathrm{T}_{1} \mathrm{c}_{12} \mathrm{e}_{1}+\mathrm{T}_{1} \mathrm{e}_{1}+\mathrm{c}_{11} \mathrm{e}_{1}+\mathrm{c}_{12} \mathrm{e}_{1}+\mathrm{e}_{1}=0$

Where,

$e_{1}=\xi_{2}=-\left[a_{1} \xi_{2}+2 a_{2} \xi_{2} x_{2}+\left(a_{4} x_{5}-\frac{1}{2} a_{6} x_{5} \frac{1}{\sqrt{a_{5}+a_{6} x_{4}}}\right) x_{3}^{2}+2 x_{3}\left(\xi_{3}-\mu_{1}\right)\left(a_{3}+a_{4} x_{4}-\sqrt{a_{5}+a_{6} x_{4}}\right)\right]$

By considering,

$\left\{\begin{array}{c}A=\left[a_{1} \xi_{2}+2 a_{2} \xi_{2} x_{2}+\left(a_{4} x_{5}-\frac{1}{2} a_{6} x_{5} \frac{1}{\sqrt{a_{5}+a_{6} x_{4}}}\right) x_{3}^{2}+2 x_{3}\left(\xi_{3}\right)\left(a_{3}+a_{4} x_{4}-\sqrt{a_{5}-a_{6} x_{4}}\right)\right] \\ B=T_{1} 2 x_{3}\left(a_{3}+a_{4} x_{4}-\sqrt{a_{5}+a_{6} x_{4}}\right)\end{array}\right.$

And the $\mu_{1}$ is obtained by:

$\mu_{1}=\frac{T_{1} c_{11} e_{1}+T_{1} c_{11} e_{1}+T_{1} A+c_{11} e_{1}+c_{12} e_{1}+e_{1}}{B}$

Similarly, for $\mu_{2}$ 
$\mathrm{r}_{2}=\mathrm{c}_{21} \mathrm{e}_{2}+\mathrm{e}_{2}$

Where,

$$
\left\{\begin{array}{c}
e_{2}=-x_{4}=-x_{5} \\
e_{1}=-x_{5}=-\xi_{5}-\mu_{5} \\
T_{2}\left(-c_{21} x_{5}-\xi_{5}-\mu_{2}\right)+c_{21} e_{2}=0
\end{array}\right.
$$

Finally, the control signal $\mu_{2}$ is given by:

$$
\mu_{2}=\frac{\left(-T_{2} c_{21} x_{5}+T_{2} \xi_{5}\right)+c_{21} e_{2}+e_{2}}{T_{2}}
$$

\section{SIMULATION RESULTS}

In this section, the simulations of the proposed ASC is performed and the comparative analysis of ASC and SMC performance is shown by taking altitude $=4 \mathrm{~m}$ and angle $=0.4 \mathrm{rad}$ as a reference signal.
In Fig. 3 the control inputs of the system are taken as $\mu_{1}$ and $\mu_{2}$. Initially $\mu_{1}$ starts from zero and converges to 200 after the delay of 1 second while $\mu_{1}$ starts from 410 and converges to -100 .

In Fig. 4, the reference altitude is set as $3 \mathrm{~m}$ from the ground, proposed controller follows by ASC and SMC control techniques and it is found that ASC it achieves stability just after 0.6 seconds whereas SMC almost takes 1.5 seconds for the same task.

Finally, Fig. 5, the rotational angle of the Mini Helicopter is also compared between ASC and SMC. The simulation result shows the approximate equal response for both controllers. This comparative analysis has easily acknowledged the importance of the proposed controller over SMC and other existing techniques.

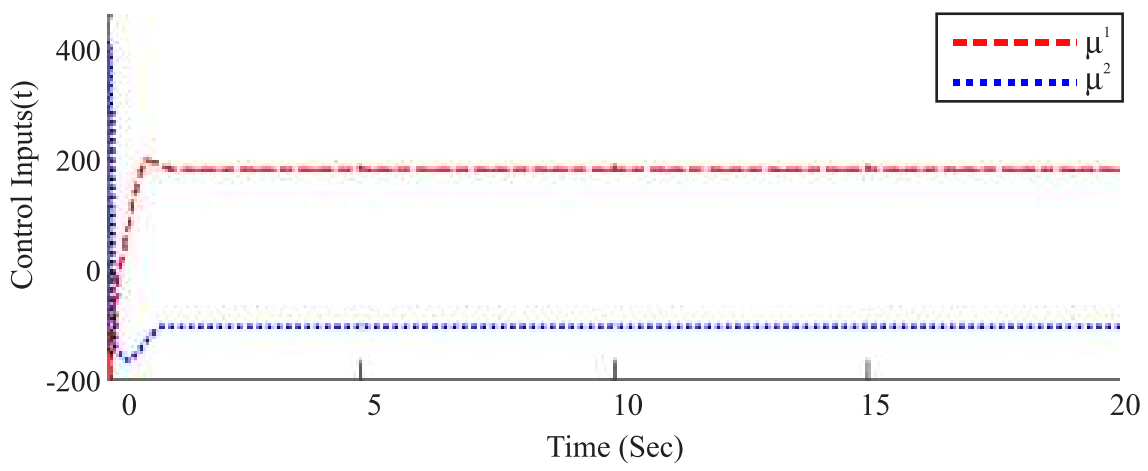

FIG. 3. CONTROL INPUTS OF THE SYSTEM

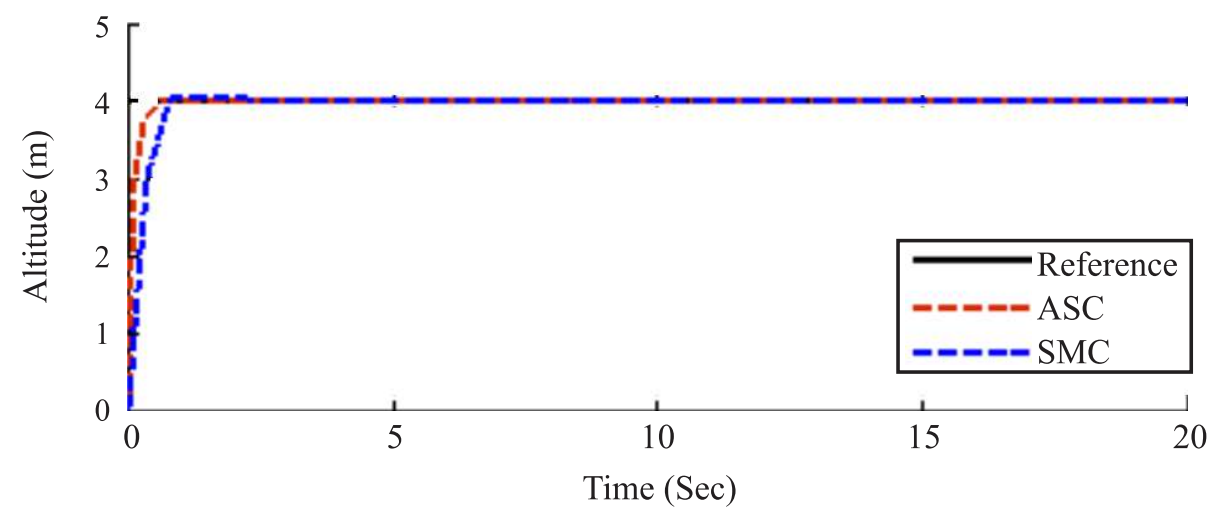

FIG. 4. COMPARISON OF ALTITUDES BETWEEN SMC AND ASC 


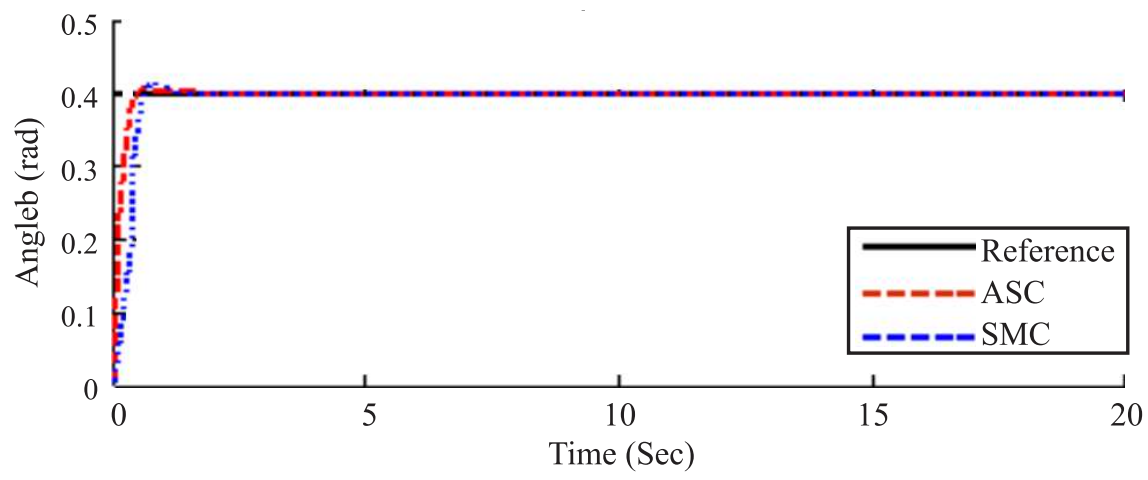

FIG. 5. COMPARISON OF ANGLES BETWEEN SMC AND ASC

\section{CONCLUSION}

In this research, a new ASC strategy is suggested for the stabilization of altitude and angle of nonlinear model of MIMO MH. ASC is used to estimate the uncertainties of the above system model and a novel ASC is designed via synergetic control theory. Moreover, the convergence rate of the above proposed approach is also investigated. Stability of the above system is proven, in sense of closed-loop bounded signals, and its tracking errors converges to zero. Simulated results validated the proposed control approach and achieved the tracking performance.

\section{ACKNOWLEDGMENT}

This work was supported in part by the National Natural Science Foundation of China under Grant 61573097 and 91748106 , in part by Key Laboratory of Integrated Automation of Process Industry (PALN201704), in part by the Fundamental Research Funds for the Central Universities (3208008401), in part by the Qing Lan Project and Six Major Top-Talent Plan, and in part by the Priority Academic Program Development of Jiangsu Higher Education Institutions.

\section{REFERENCES}

Carstens, N., "Development of a Low-Cost, Low-Weight Flight Control System for an Electrically Powered ModelHelicopter", Ph.D. Thesis, University of Stellenbosch, Stellenbosch, 2005.
[2] Valavanis, K.P., (Editor), ”Advances in Unmanned Aerial Vehicles: State-of-the-Art and the Road to autonomy”, Science \& Business Media, Volume 33, Springer, 2008.

[3] Sanchez-Lopez, Luis, J., Wang, M., Miguel, A., OlivaresMendez, M.M., and Holger, V., "A Real-Time 3D Path Planning Solution for Collision-Free Navigation of Multirotor Aerial Robots in Dynamic Environments", Journal of Intelligent \& Robotic Systems, pp. 1-21, 2018.

[4] Samir, D.,"Design and Control of Quadrotors with Application to Autonomous Flying”, Thesis LIB, Epfl, 2007.

[5] McRuer, D.T., Graham, D., and Ashkenas, I., "Aircraft Dynamics and Automatic Control", Volume 740, Princeton University Press, 2014.

[6] Calise, A.J., and Rolf, T.R., "Nonlinear Adaptive Flight Control Using Neural Networks", IEEE Control Systems, No. 6 pp. 14-25, 1998.

[7] Padfield, G.D., "Helicopter Flight Dynamics: The Theory and Application of Flying Qualities and Simulation Modelling”, John Wiley \& Sons, 2008.

[8] Ali, H.I., Noor, S.B.B.M., Bashi, S.M., and Marhaban, M.H., "A Review of Pneumatic Actuators (Modeling and Control)", Australian Journal of Basic and Applied Sciences, Volume 3, No. 2, pp. 440-454, 2009.

[9] Safwan, M., Uddin, V., and Asif, M., "Nonholonomic Mobile Robot Trajectory Tracking Using Hybrid Controller", Mehran University Research Journal of Engineering and Technology, Volume 35, No. 2, pp, 161-170, Jamshoro, Pakistan, April, 2016. 
[10] Slotine, J.-J.E., and Li, W.,"Applied Nonlinear Control", Volume 199, No. 1, Englewood Cliffs, NJ Prentice Hall, 1991.

[11] De Queiroz, M.S., Darren, M.D., Siddharth, P.N., and Fumin, Z., "Lyapunov-Based Control of Mechanical Systems", Science \& Business Media, Springer, 2012.

[12] Maass, W., Thomas, N., and Henry, M., "Real-Time Computing without Stable States: A New Framework for Neural Computation Based on Perturbations", Neural Computation, Volume 14, No. 11, pp. 2531-2560, 2002.

[13] Mutambara, and Arthur, G.O., "Design and Analysis of Control Systems", CRC Press, 2017.

[14] Neilson, P.D., and Megan, D.N., “An Overview of Adaptive Model Theory: Solving the Problems of Redundancy, Resources, and Nonlinear Interactions in Human Movement Control", Journal of Neural Engineering, Volume 2, No. 3, pp. S279, 2005.

[15] Sakthivel, T., and Venkatesan, C., "Rotorcraft Control Response Using Linearized and Non-Linear Flight Dynamic Models with Different Inflow Models", The Aeronautical Journal, Volume 121, No. 1238, pp. 553-575, 2017.

[16] Pallett, T.J., and Ahmad, S., "Real-Time Helicopter Flight Control: Modelling and Control by Linearization and Neural networks", 1991.

[17] Sira, R.H., Zribi, M., and Ahmad, S., "Dynamical Sliding Mode Control Approach for Vertical Flight Regulation in Helicopters", IEE Proceedings on Control Theory and Applications, Volume 141, No. 1, pp. 19-24, 1994.

[18] Kaloust, J., Ham, C., and Qu, Z., "Nonlinear Autopilot Control Design for a 2-DOF Helicopter Model", IEE Proceedings on Control Theory and Applications, Volume 144, No. 6, pp. 612-616, 1997.

[19] Shima, T., Moshe, I., and Oded, M.G., "Sliding-Mode Control for Integrated Missile Autopilot Guidance", Journal of Guidance, Control, and Dynamics, Volume 29, No. 2, pp, 250-260, 2006.

[20] Åström, K.J., and Björn, W., "Adaptive Control”, Courier Corporation, 2013
[21] Todorov, E., and Michael, I.J., "Optimal Feedback Control as a Theory of Motor Coordination", Nature Neuroscience, Volume 5, No. 11, pp, 1226, 2002.

[22] Latash, M.L., John, P.S., and Gregor, S., "Toward a New Theory of Motor Synergies", Motor Control, Volume 11, No. 3, pp. 276-308, 2007.

[23] Nechadi, E., Harmas, M.N., Hamzaoui, A., and Essounbouli, N., "Type-2 Fuzzy Based Adaptive Synergetic Power System Control", Electric Power Systems Research, Volume 88, pp. 9-15, 2012.

[24] Kimon, V.P., (Editor), "Advances in Unmanned Aerial Vehicles: State-of-the-Art and the Road to Autonomy", Science \& Business Media, Volume 33, Springer, 2008.

[25] Awelewa, A.A., "Development of Nonlinear Control Schemes for Electric Power System Stabilization", Ph.D., Thesis, Covenant University, Nigeria, 2016.

[26] Richard. K., "Approximating Innovation Potential with Neurofuzzy Robust Model", InvestigacionesEuropeas de Dirección y Economía de la Empresa, Volume 21, No. 1, pp. 35-46, 2015.

[27] Saima, H., Khanesar, M.A., Kayacan, E., Jaafar, J., and Khosravi, A., "Optimal Design of Adaptive Type-2 Neuro-Fuzzy Systems: A Review", Applied Soft Computing, Volume 44, pp. 134-143, 2016.

[28] Slotine, J.-J.E., and Coetsee, J.A., “Adaptive Sliding Controller Synthesis for Nonlinear Systems", International Journal of Control, Volume 43, No. 6 , pp. 1631-1651, 1986.

[29] Zhenhua, J., and Dougal, R.A., "Synergetic Control of Power Converters for Pulse Current Charging of Advanced Batteries from a Fuel Cell Power Source", IEEE Transactions on Power Electronics, Volume 19, No. 4, pp. 1140-1150, 2004.

[30] Yu, Z., Jiang, Z., and Yu, X., "Indirect Field-Oriented Control of Induction Machines Based on Synergetic Control Theory", 21st Century IEEE Power and Energy Society General Meeting Conversion and Delivery of Electrical Energy, pp. 1-7, 2008. 